\title{
GAIA Level 1 Intrapartum Stillbirth
}

National Cancer Institute

\section{Source}

National Cancer Institute. GAIA Level 1 Intrapartum Stillbirth. NCI Thesaurus. Code C128022.

GAIA Level 1 Intrapartum Stillbirth is defined by four criteria: first, delivery of an infant with no of signs of life at birth, including the following: no spontaneous movements, no umbilical cord pulse, no heartbeat, no respirations, and an Apgar score of 0 at both 1 and 5 minutes. Determination of the absence of signs of life is made by physical examination after delivery, with or without electronic monitoring of heart rate, respiratory rate, and pulse oximetry; second, evidence of a live fetus prior to the onset of labor (confirmed by documentation of fetal movement and of fetal heart tones by ultrasound prior to onset of labor). NOTE: In the absence of evidence of a live fetus prior to the onset of labor, the fetal death should be reported as a stillbirth or an antepartum stillbirth; third, attended delivery followed by post-delivery physical examination by an obstetrician, a neonatologist, a pediatrician, a maternal-fetal medicine specialist, or a pathologist that is consistent with intrapartum death. In a setting where access to a specialist is not feasible, diagnosis by a health care provider trained or experienced to make the diagnosis is acceptable (e.g. general practice physician, mid-wife, or other qualified trained practitioner); fourth, gestational age within the pre-defined range for selected stillbirth definition as assessed by maternal and/or fetal-neonatal parameters (level 1 in GA assessment algorithm). 